\title{
The Potential of Mean Force concept for bridging (length and time) scales in the modeling of complex porous materials
}

\author{
Katerina Ioannidou ${ }^{1,2,3}$, Benoit Carrier $^{4}$, Matthieu Vandamme ${ }^{4}$, and Roland Pellenq ${ }^{1,2,3,}$ \\ ${ }^{1}$ Department of Civil and Environmental Engineering, Massachusetts Institute of Technology (MIT), Cambridge, MA, USA \\ ${ }^{2}$ Centre Interdisciplinaire de Nanosciences de Marseille, Aix-Marseille Université, CNRS, Campus de Luminy, Marseille, France \\ ${ }^{3}<M S E>^{2}$ The joint CNRS / MIT / Aix-Marseille Université laboratory, MIT, Cambridge, MA 02139, USA \\ ${ }^{4}$ Laboratoire Navier, Université Paris-Est, CNRS, ENPC, IFSTTAR, Marne-la-Vallée, France
}

\begin{abstract}
We introduce the concept of Potential of Mean Force, PMF, as a way to implement upscaling modeling from the nano-scale to micron-scale. A PMF is a free energy function representing in an effective way the interactions between objects (cement hydrates, clay platelets, etc.) at thermodynamics conditions. The PMF is therefore the key piece of information allowing to coarse-grained Physical-Chemistry information in a meso-scale model formulation. The use of PMF offers a huge computational advantage as it allows a straight up-scaling to the meso-scale while keeping essential interactions information that are the hallmark of Physical-Chemistry processes. Such a coarse-grained modeling integrates atomistic response into inter-particle potentials that fully propagate molecular scale information all the way to the meso-scale.
\end{abstract}

\section{Introduction}

In this paper, we describe the concept of Potential of Mean Force as the tool for modeling soft and hard amorphous porous materials across length and time scales. This Bottom-Up approach integrates different scales of knowledge into accurate models of relevant phenomena and complex systems across multiple length and time scales. We outline the originality of the method, its basic ingredient, and give two examples of applications, namely cement and clays minerals. We believe that this approach provides a unique opportunity for developing a full predictive modeling and simulations for amorphous complex porous materials from molecular to engineering scales. Besides a few examples such a zeolites, the vast majority of porous materials are multi-scale disordered complex systems. They are usually classified according to their characteristic pore size $\varnothing$ : microporous solids have $\varnothing$ lower than $2 \mathrm{~nm}$, mesoporous $2 \mathrm{~nm}<\varnothing<20 \mathrm{~nm}$ and macroporous $\emptyset>20 \mathrm{~nm}$. Such materials are ubiquitous on Earth, be it in the Earth Crust (clays, porous carbons, zeolites, serpentines rocks, asphalt, wood etc.) or manmade (concrete, carbons, gas storing porous materials, nuclear fuels, etc.). While macroscopic porous media theory has a long tradition in all fields of engineering, ranging from Civil, Environmental, Chemical, Biochemical, many current and future challenges in the field of energy and environment heavily rely on breakthroughs in our understanding of the physics and mechanics of the intimate interplay between the two ingredients involved in the make up of such porous materials: their complex solid texture that results often from an initial soup chemistry and the role of liquids confined to their pore voids; all of which can be gathered under the term Poromechanics when it come to understanding the link between Physical-Chemistry and Mechanics.
Cement and clays minerals are salient examples of multiscale porous materials. While clays is obvious the important natural materials in Soil Science and Agriculture, cement is widely produced more than any other synthetic material on Earth. The current worldwide cement production stands at three billion tons, enough to produce more than twenty billion tons or one cubic meter of concrete per capita per year. There is no other material that can replace cement in the foreseeable future to meet our societies' needs for housing, shelter and infrastructure by the unique property of cement transforming from liquid to stone at room temperature. Nevertheless cement faces an uncertain future, due to a non-negligible ecological footprint that amounts to 5$10 \%$ of the worldwide $\mathrm{CO}_{2}$ production. On the other hand, thanks to breakthroughs in science and engineering, cement and concrete have a novel potential to contribute to a sustainable development encompassing economic growth, social progress while minimizing on the ecological footprint. Manufacturers are willing to produce high-performance concrete that is not only environmentally more efficient (consuming less energy and giving off less $\mathrm{CO}_{2}$ ) but qualitatively transformed. The recent disasters of the Japanese nuclear plant of Fukushima (with the explosion of the reactor's concrete dome) and the oil spill in the Gulf of Mexico (due to an uncontrolled cement liner setting during well completion) highlight the lack of materials technology and the need of renewing engineering practices from science-based principles. A similar yet less dramatic lack of knowledge expands into many fields of environmental and energy related materials research, from the environmental compatibility of shale-gas or nuclear wastes storage (for which a thorough understanding of the interaction between cement and clay remains to be accomplished. Refreshingly, concrete starts to achieve its integrity through research and craftsmanship - much like any other material. This requires a holistic approach in which progress comes from the nano-scale and is featured into innovative structural engineering 
applications whose impact on sustainable development can be evaluated with advanced environmentaleconometric impact studies.

Up to now, innovation in the field of cementitious and clay materials was a classical top-down approach where a problem at one scale was solved at the scale just below. This was somewhat successful to address mechanical properties but never addressed environmental issues up front. Today the advancement of fundamental knowledge leans toward developing a predictive BottomUp approach from the electronic and atomic level to micro-texture and macroscopic engineering properties. In the particular case of the cement paste, the first outcome of this approach was a realistic and consistent atomistic model of cement hydrates (see Fig. 1a), deciphering the "DNA of cement". Since then, progresses have been made on many fronts: in particular the realistic micron-scale model (see Fig. 1b) opens unprecedented possibilities and constitutes the starting point for modeling fracture, creep and aging of the cement paste in the context of durability that is the core of the present paper. A new multi-scale approach is needed to integrate different scales of knowledge into accurate models of relevant phenomena and complex systems across multiple length and time scales, ultimately allowing for the development of concept materials with improved or new properties. We propose a shift of paradigm based on a bottom-up approach that can be applied to and tested for most important technological, economical and environmental materials: cement, soils and sediments, ceramics, solid nuclear fuels, geo-materials, geo-polymers, rocks, organic minerals, organic-inorganic composites, biological tissue; all being important classes of multi-scale materials.
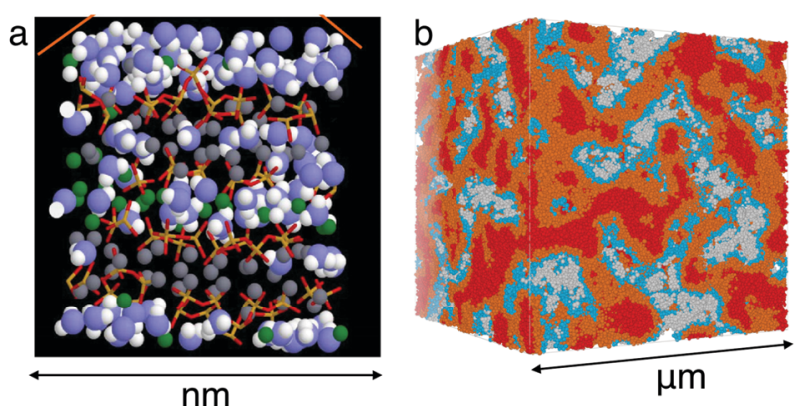

Figure 1: a) At the root of the bottom-up approach the first atomistic model of cement [1]. b) Up-scaling to the first mesoscale model of cement hydrates [2]. The color code of the meso-scale model indicates locally dense and loose regions of matter (the density increases from gray, blue, orange to red).

The bottom-up approach that we propose is amenable to bridge time and length scales based on the handshake between numerical simulations and experiments. Ultimately, this approach should be transformational, shifting existing technology from empirical practice to science-based knowledge in the context of sustainability, durability, energy and waste management with the emergence of a new interdisciplinary field of research at the frontier between Sciences and Engineering. In the proposed approach, novel simulation methods from the large toolbox of computational soft matter and glass physics can be combined to enable science-based engineering of new materials.

\section{The Potential of Mean Force Concept at the core of the Bottom-Up Approach}

\subsection{Atomistic calculations}

Our starting point is the atomic structure (of cement hydrates or clays, ceramics, etc.) from which, using state-of-the art inter-atomic potentials, one can derive the so-called "Potential of Mean Force" (PMF) between different phases using the perturbation approach from Statistical Physics for free energy calculations. A PMF is a free energy function representing in an effective way the interactions between objects (cement hydrates, clay platelets, etc.) at a given temperature. The PMF is therefore the key piece of information allowing to coarse-grained Physical-Chemistry information in a meso-scale model formulation. The use of PMF offers a huge computational advantage as it allows a straight upscaling to the meso-scale while keeping essential interactions information that are the hallmark of Physical-Chemistry processes. Such a coarse-grained modeling integrates atomistic response into inter-particle potentials that fully describe the atomic interactions and enables to reach much larger time and length scales. We thus propose to use the PMF coarse-grained colloidal modeling approach to translate the understanding at atomistic scales to the scale of micro-texture for amorphous porous materials.

In the recent years, we have elaborated further the idea and developed new meso-scale colloidal model of cement hydrates (in the hardened cement paste) and clay minerals. In both cases, the complex pore network and the structural heterogeneities naturally emerge from the short-range cohesive interactions typical of nano-scale grains of cement hydrates and nano-platelets of clay minerals and from non-equilibrium conditions. The key point of this approach is that if the coarse-graining unit is well represented, the access to the physics depends only on the appropriate level of modeling the interactions. The simulation techniques at the colloidal scale are the same as the ones used at the atomistic scale but applied to grains of matter containing thousands of atoms or molecules. These are Molecular Dynamics (MD) and Monte Carlo (MC) in different statistical ensembles. New scheme combining MD and Grand Canonical Monte Carlo (GCMC) have been applied with success to simulate the early and late stages of cement hydration, imitating the precipitation of cement particles [2-5] and the sedimentation of laponite clay mineral [6]. The bottom-up approach therefore provides a rigorous link between the physical chemistry of materials and their overall functional and mechanical properties including elasticity, strength, fracture and permeability, etc...

It opens the route to the engineering scale by providing the relevant scale for analytical coarse-graining and 
homogenization of the properties. At the micron-scale, the texture has either the features of a granular material, with well-defined particles of different shapes and sizes and cohesive-frictional contact interactions embodied in a solid or liquid phase, or the features of a porous aggregate. The mechanical properties at this scale involve thus granular-type disorder and multi-phase coupling, which can be more efficiently addressed by using discrete-element modeling (DEM) or semicontinuum approaches such as Lattice Element Methods (LEM) or Material Point Models (MPM) possibly coupled with a liquid phase or a binding solid phase; see for example Ref. 6 . The method starts with the atomistic calculations of the change in interaction free energy as a function of the separation distance between two nanograins at the atomistic scale using a thermodynamics integration scheme based on a perturbation theory appraoch. Note that the integration constant is usually set to zero. Then the Gay-Berne potential type for oblates particles, including platelet-shaped object, can be fitted to the PMF at the meso-scale. Note that the Gay-Berne potential reduces into the well-known Lennard-Jones form for spherical grains). The GB potential is defined as:

$$
U=4 \varepsilon\left\{\left(\frac{\sigma}{h_{12}+\sigma}\right)^{12}-\left(\frac{\sigma}{h_{12}+\sigma}\right)^{6}\right\} \eta_{12} \chi_{12}
$$

where $\varepsilon=1$ determines the energy scale, $\sigma$ is the atomic interaction radius and $h_{12}$ is a function that approximates the anisotropic interparticle distance. $\eta_{12}$ and $\chi_{12}$ characterize anisotropic interactions of particles due to their shapes and relative orientations. In the case of clay platelets, the PMF for face-to-face interactions between layers following the procedure of Ebrahimi et al. [7], is given in Figure 2. It represents the change in system free energy at $300 \mathrm{~K}$ as a function of the distance between layer and is given as an energy per surface area hence allowing the scaling as a function of the particle size. This function shows 3 minima that indeed correspond to the known stability distances for sodium Montmorillonite. The PMF is then taken as an effective interaction between particle of given sizes and allows implementing an efficient mesoscale modeling scheme as the atomic details (water content, ions density etc...) are gathered in the PMF function. It worth noting that extracting a PMF from atomistic scale is computationally expensive and that it si remains a state function that is only valid for the thermodynamic conditions ( $\mathrm{P}, \mathrm{T}$, ion concentration, water content...) that were used to establish it. This is clearly a drawback of the approach and other less expensive schemes have to be explored (see below). The fit of the PMF for sodium Montmorillonite extracted from atomistic scale requires going beyond the Gay-Berne potential that can only describe a single well PMF. In the case of cement hydrate $(\mathrm{CSH})$, extracting the PMF is more difficult as $\mathrm{CSH}$ is not a well defined compound with a weill defined chemistry but rather a non stoechimetric coumpound with a range of possible compositions
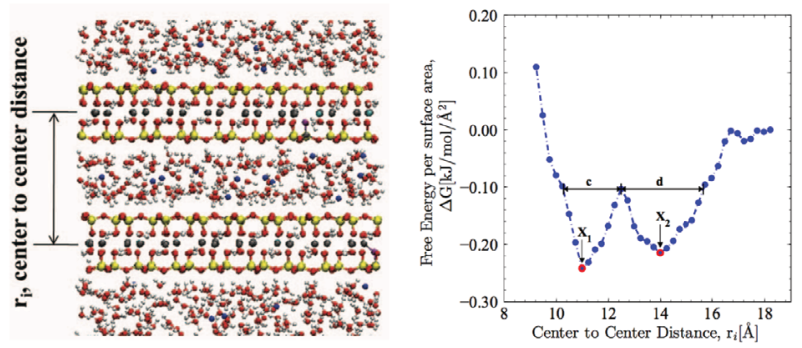

Figure 2: Face-to-face PMF for Sodium-Montmorillonite clay at $300 \mathrm{~K}[7]$

measured in terms of the $\mathrm{Ca} / \mathrm{Si}$ ratio spanning the range from 1 to 2 . $\mathrm{CSH}$ is hovever a (defective) layered material for $\mathrm{Ca} / \mathrm{Si}$ ratio retainting the layered structure of a parent mineral called Tobermorite. In Figure 3, we give the PMF extracting from atomistic scales for the $\mathrm{Ca} / \mathrm{Si}=1.65$ (that is the most common/probable value of $\mathrm{CSH}$ in a real cement paste [8]. PMF was recently obtained for tobermorite [9] $(\mathrm{Ca} / \mathrm{Si}=1)$ predicting essentially a single well potential behaviour. Again, as for clay Montmorillonite, CSH-PMF cannot be stricly fitted to GB potential. A better description can be obtained using a 12-24 Lennard Jones together with a Yukawa term to account for the repulsive shoulder that is observes at larger distances [3-5].

Validation of the PMF bottom-up approach came through the development of advanced analytical tools to compute quantities that are measured in experiments at the scale well below the micron, such as scattering intensity, pore size distribution, surface area, local volume fractions, indentation modulus and hardness. All these quantities that were "measured" in the simulations and compared successfully with experiments provided the first consistent characterization of the complex and elusive meso-scale structure of cement [2]. The experimental tools applied to measure such quantities in cement were electron-microscopy imaging, nanoindentation tests, small angle X-rays and neutron scattering (SAXS and SANS), nuclear magnetic resonance (NMR) spectroscopy and adsorption desorption experiment of $\mathrm{N}_{2}$ and water. A remarkable agreement with experiment was achieved for both cement and clays [2,7].

\subsection{Improving Primitive model predictions}

The cohesion and stability of such charged layers separated by a solvent containing counterions has long been discussed in the framework of the DerjaguinLandau-Verwey-Overbeek (DLVO) theory. The DLVO theory is the combination of the repulsive electrical double layer interaction with the attractive van der Waals interactions [10]. The repulsive electrostatic interaction is obtained by solving analytically the PoissonBoltzmann equation of a system made of positive point 

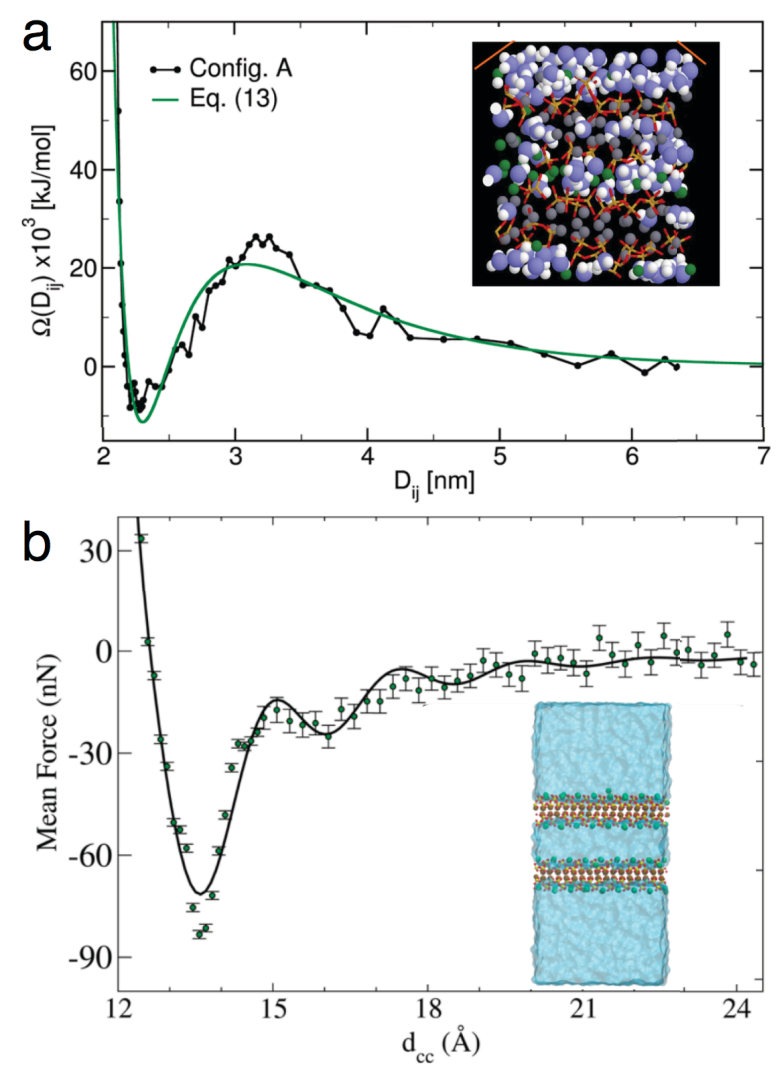

Figure 3: a) Face-to-face $\mathrm{PMF}$ for $\mathrm{CSH}$ for $\mathrm{Ca} / \mathrm{Si}=1.65$ at $300 \mathrm{~K}$ Black line, atomistic simulation (Ref), green line the fit using a Yukawa LJ-(12-24) form [8]. b) Face-to-face PMF for CSH for Tobermorite, $\mathrm{Ca} / \mathrm{Si}=1$ at $300 \mathrm{~K}[9]$

charges between negatively charged surfaces. Making use of the Poisson-Boltzmann equation, the electrostatic pressure $P$ between two charged surfaces in function of the surface separation $D$ is given by:

$$
P(D)=k_{B} T\left(\rho_{0}(D)-\rho_{0}(\infty)\right)
$$

where $k_{B}$ is the Boltzmann constant, $T$ the temperature and $\rho_{0}$ is the cation concentration at the mid-plane for a surface separation $x$. This pressure depends only on the increase on the ionic concentration at the center of the pore and therefore its origin is entropic. However, it is now known that a system of charged plates and counterions can generate strong attractive electrostatic interactions, the so called ionic correlation forces [10].

To study such systems numerically, the so-called Primitive Model wad introdiced in the 80's. In the Primitive Model, the charged lamellae and the hydrated cations are represented by uniformly charged infinite planar surfaces and by charged hard spheres, respectively, while the solvent is represented by a dielectric continuum. Monte Carlo simulations in the framework of the Primitive Model showed that fluctuations of the ionic concentration in the interlayer space result in an instantaneous polarization and give rise to an attractive ionic fluctuation interaction, the socalled ionic correlations forces. Thus, the behavior of the system is governed by a competition between repulsive entropic forces and attractive energetic forces. For divalent or trivalent cations, the attractive energetic contribution can exceed the repulsive entropic component of the pressure and results in one or several attractive wells of the pressure between the lamellae. Figure 5 shows the pressure between two montmorillonite layers in function of the distance between the layers, as predicted by the Primitive Model. Two cases are considered: a montmorillonite with sodium counterions and a montmorillonite with calcium counterions. As predicted by the Poisson-Boltzmann equation, in the case of sodium counterions, the pressure is always repulsive reflecting the fact that it is a meanfield solution ignoring thermally activated ion density

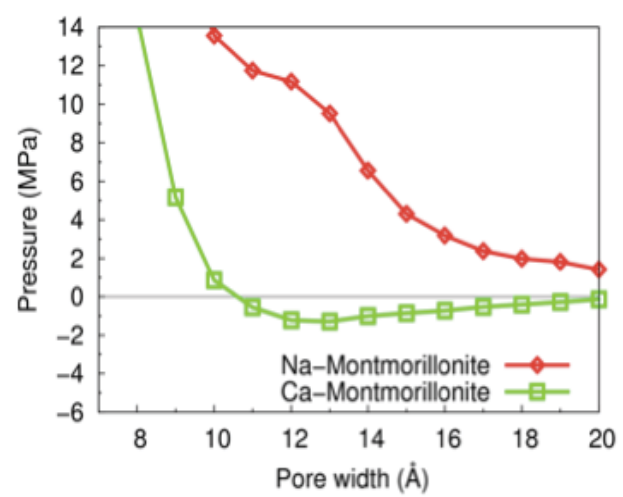

Figure 4: Interlayer pressure computed with the Primitive Model versus pore width for montmorillonite with sodium (red diamond symbols) or calcium (green square symbols) interlayer cations [11].

fluctuations. In contrast, in the case of calcium counterions, attractive pressures are observed for pore sizes larger than $10 \AA$ and explains the experimental observation that exfoliating completely calcium montmorillonite layers is difficult. Note that the corresponding PMF is simply the integral form of the pressure curve along the inter particle direction. The Primitive Model has obviously its own limitations. The surfaces of the slit pore are assumed to be flat and uniformly charged, the ions are supposed to be fully hydrated and mobile and the solvent is considered to be a dielectric continuum the dielectric constant of which is the one of bulk water. These hypotheses make the Primitive Model questionable, in particular at low surface separation. In this case, the molecular structures of the solvent and the surfaces have to be taken into account. Therefore, numerous works adopted a fullatomistic description of the system to study for instance the intralamellar swelling of smectite clays [7]. However, as mentioned above, the computational cost of these atomistic simulations is very significant preampting coarse-grained simulations. Accordingly, it is necessary to determine more precisely the complexity which needs to be introduced into the models of lamellar systems and the key physical ingredients responsible for the cohesion of such materials. Moreover, the link between the Primitive Model and the full-atomistic model has never been made clear until the $\mathrm{PhD}$ work of Carrier in 2013 [11].. 
It is crucial to determine what level of complexity is required in the modeling of the system in order to get meaningful results, depending on the charge of the surfaces, on the valency of the cations, and on the separation between the charged surfaces. In particular, we want to answer the following questions: is the Primitive Model sufficient to study the effect of confined electrolytes on the cohesion of the system, especially when the pore width is small? At which critical value of the so-called electrostatic coupling do the fluctuations of the ionic concentrations have to be considered? The electrostatic coupling is defined as the ratio between the interaction energy between a counterion and a charged surface and the thermal energy, and is therefore proportional to the product of the counterion valency and the surface charge. To address these questions, we present computer simulations of confined electrolytes between charged clay layers. In addition to the DLVO theory, we used various models of increasing complexity: the Primitive Model (PM), the Explicit Solvent Primitive Model (ESPM) and the full-atomistic model. The Explicit Solvent Primitive Model is a model we propose to take into account explicitly the solvent while keeping the simplicity of the Primitive Model. As in the Primitive Model, in the ESPM model the charged lamellae are represented by infinite uniformly charged planes. The counterions and the solvent particles are represented by point charges and point dipoles, respectively, embedded in soft spheres; solvent molecules being decored with a dipole moment whose norm is adjusted to reproduce bulk water dielectric constant at room temperature. A dielectric constant equal to 78.5 was obtained with a dipole moment equal to 1.7 D. This value was lower than the experimental dipole moment of water molecules in a liquid phase (i.e. about 2.6 D). Indeed, this simplistic model is not detailed enough to capture the dielectric response of water nor the real hydrogen bond governed structure of liquid water. Figure 6 shows the interaction pressure between layers of montmorillonite versus pore width either with $\mathrm{Na}$ interlayer cations or with $\mathrm{Ca}$ interlayer cations (calculated from the virial definition in the the direction perpendicular to the layers). It compares the results from the full-atomistic appraoch with the prediction of the ESPM model. The quatitative agreement between the full-atomistic model and the ESPM model suggests that the ESPM model is able to capture the relevant physical mechanisms governing the cohesion of charged layers in a solvent, for a much lower computational cost than fullatomistic models (i.e. the same accuracy could be obtained about one order of magnitude faster in terms of computational cost). The results of the ESPM model contrast with the ones of the Primitive Model. Indeed the repulsive pressure predicted by the Primitive Model at any pore size in the case of Na-montmorillonite is invalidated by the ESPM model. Therefore, an explicit solvent proves to be critical to explain the cohesion of charged lamellae. The cohesion of Na-montmorillonite can be explained neither with the mean-field DLVO theory nor with the Primitive Model. According to the Primitive Model, the entropic repulsion is always greater
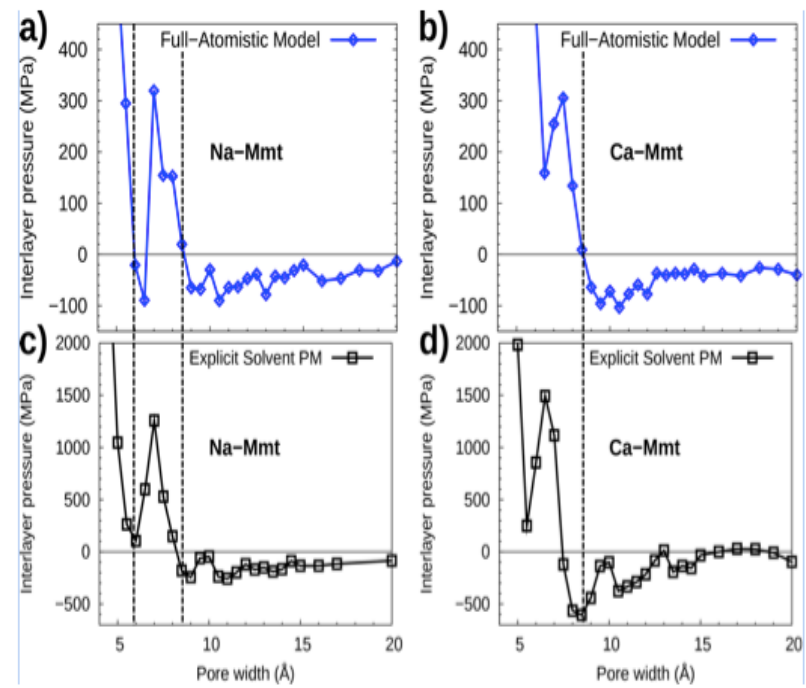

Figure 5: Interlayer pressure versus pore width for ( $\mathrm{a}$ and $\mathrm{b}$ ) Na-Montmorillonite and for (c and d) Ca-Montmorillonite computed with (a and c) the full-atomistic model or with (b and d) the Explicit Solvent Primitive Model. [11].

than the electrostatic attraction due to ionic fluctuations. However, the assumption that the dielectric constant of the confined fluid is equal to the dielectric constant of bulk water leads to a significant underestimation of the energetic attractive contribution. The ESPM model, as well as the full-atomistic model, showed that with sodium interlayer cations, stable states and attractive pressure wells exist no matter the surface charge, while the results of the Primitive Model showed that for the same systems the interaction pressure was always repulsive. Therefore, the solvent plays an important role in the cohesive behavior of the system. The effect of the solvent cannot be captured by a simple dielectric continuum as in the Primitive Model and the solvent has to be taken into account explicitly. Moreover, the results of the ESPM model suggest that ionic correlation forces play a significant role even at low electrostatic couplings.

\section{Conclusion}

We introduced the concept of Potential of Mean Force (PMF) as a tool to implement up-scaling simulations from the nano-scale to the micron-scale reaching out to so-called engineering scales in the cases of cement and clay minerals. There are different levels of calculations that can be performed to extract the PMF. The most computationally expensive and most accurate is certainly to use an atomistic description together with a thermodynamic integration route (perturbation theory approach).

The interaction between charged objects immersed in an electrolyte such as cement hydrates or clay particles has long been studied using colloidal theories such as DLVO, ionic correlation forces, etc... These are easier ways to extract PMFs between particles using a direct integration scheme at a much smaller computational expense. However, these colloidal approaches can be 
seen as over-simplistic as they might not give even a qualitative account of experiments in terms of the stability and cohesion of these charged objects mainly because of a poor description of the confined solvent. Here we showed that taking into account the solvent explicitly is necessary to describe the cohesion of charged lamellar systems. Indeed, the solvent exhibits a complex dielectric response that impacts strongly the electrostatic interactions in the system. In order to keep the simplicity of the Primitive Model while modeling water explicitly, we introduce the Explicit Solvent Primitive Model, in which each water molecule is modeled by a point dipole. The ESPM model gives results that are in good agreement with the results of the full-atomistic model, but at a lower computational cost (i.e. approximately one order of magnitude faster). We recommend to use the ESPM model to compute interactions between charged grains and extract PMFs at the colloidal scale.

This approach further gives the foundation of meso-scale investigations of fracture and creep of complex systems such as cement and clays. Long time scale is crucial for investigating creep, the slow drifting of the nano-scale cement or clay units when under stress. Large length scales are equally critical in order to understand longrange space correlations and to obtain microscopic features when investigating fracture.

This work was supported by the ICoME2 Labex (ANR-11LABX-0053) and the Aix-Marseille University AMIDEX foundation.

\section{References}

1. Pellenq et al., Proc. Natl. Acad. Sci. USA 106, 16102-1610 (2009)

2. K. Ioannidou, K. J. Krakowiak, M. Bauchy, C. G. Hoover, E. Masoero, S. Yip, F.-J. Ulm, P. Levitz, R. J.-M. Pellenq, and E. Del Gado, Proceedings of National Academy of Science USA, 113 (8), 2029-2034 (2016)

3. K. Ioannidou, R. J.-M. Pellenq and E. Del Gado, Soft Matter, 10, 1121-1133 (2014)

4. K. Ioannidou, M. Kanduc, L. Li, J. Dobnikar, D. Frenkel and E. Del Gado, The crucial effect of early-stage gelation on the mechanical properties of cement hydrates, Nature Communications, 7, 12106 (2016)

5. E. Del Gado, K. Ioannidou, E. Masoero, A. Baronnet, R. J.-M. Pellenq, F.-J. Ulm, and S. Yip, Eur. Phys. J. - Spec. Top. 223, 2285-2295 (2014)

6. F. Radjai and F. Dubois, (Wiley-Iste, Berlin), ISBN 978-1-84821-260-2 (2011)

7. D. Ebrahimi, A. Whittle, J. and R. J.-M. Pellenq, J. Chem. Phys., 140, 154309 (2014)

8. P. A. Bonnaud, C. Labbez R. Miura et al, Nanoscale 8, 4160-72 (2016)
9. S. Masoumi, H. Valipour, M. J. Abdolhosseini Qomi, J. Phys. Chem. C, 121, 5565-5572 (2017)

10. J. Israelachvili, Intermolecular and Surface Forces (Third Ed.) ISBN: 978-0-12-375182-9 (2011)

11. B. Carrier, PhD thesis, Ecole Nationale de Ponts et Chaussées, Paris (in English) 